\title{
Identification of Dickeya zeae as a Causal Agent of Bacterial Soft Rot in Banana in China
}

Jingxin Zhang, Huifang Shen, Xiaoming Pu, and Birun Lin, Key Laboratory of New Technique for Plant Protection in Guangdong, Institute of Plant Protection, Guangdong Academy of Agricultural Sciences, Guangzhou 510640, China; and John Hu, College of Tropical Agriculture and Human Resources, University of Hawaii, Honolulu, HI 96822, USA

\begin{abstract}
Zhang, J., Shen, H., Pu, X., Lin, B., and Hu, J. 2014. Identification of Dickeya zeae as a causal agent of bacterial soft rot in banana in China. Plant Dis. 98:436-442.

Bacterial soft rot of banana was first noticed in 2009 in Guangzhou city, China. The disease also was observed on various banana cultivars of different genotypes in several other cities. Symptoms of the disease included leaf wilting, collapse of pseudostems, and unusual odor. Five isolated strains that fulfilled Koch's postulates were used for biochemical testing. The five strains were most similar to Dickeya dadantii or $D$. $z e a e$, but were much less similar to $D$. paradisiaca when using several phenotype characteristics. Sequence analysis of 16S rDNA, dnaX, $\operatorname{gryB}$, and $\operatorname{rec} A$ of a reference strain revealed a similarity of $99 \%$ with

the sequences of D. zeae, rather than D. paradisiaca. Phylogenic analysis of concatenated sequences of $\operatorname{dnaX}$, gryB , and recA indicated that the banana strain constituted a distinguishable clade with several $D$. zeae strains involving rice pathogens D. zeae EC1 and ZJU1202 from Guangdong province, but the banana pathogen had several characteristics that distinguished it from the rice pathogens. Therefore, the banana pathogen was determined to be D. zeae. This is the first report of banana soft rot caused by D. zeae in China; however, the pathogen can infect other important crops.
\end{abstract}

The herbaceous, flowering banana (Musa spp.) plant is native to tropical Asia. It has been widely domesticated in at least 107 countries for its fruit, fiber, wine, and ornamental uses, and is the fourth most cultivated food crop in the world after rice, wheat, and maize. Until 2007, the area planted to banana was approximately 4.4 million ha with an output of more than 80.0 million metric tons. In south China, banana is also one of the most important economic fruits, with banana plantations encompassing more than 0.3 million ha annually. Banana plays an increasingly important role in the tropical economic regions of China, including Guangdong, Guangxi, Hainan, and Fujian provinces.

In September 2009, there was a bacterial soft rot outbreak in banana in Guangzhou, China, resulting in $82 \%$ yield losses in 10 ha of surveyed fields (17). Soft rot of banana has been reported to be caused by Pectobacterium carotovora (Erwinia carotovora) (20) in Korea, Iran, and India $(1,6,26)$. However, one symptom caused by $P$. carotovora is stunted growth (28), which was different from the symptoms observed in Guangzhou. In this outbreak, the first disease symptom observed was yellowing of petioles on emerging leaves, followed by the collapse of petioles, leaf wilting, and then death. The vascular tissue of the pseudostem of infected banana turned brown or black, and a bacterial ooze was observed on the pseudostem and carpopodium (Fig. 1). Isolates of Dickeya sp. were

Corresponding author: Birun Lin, E-mail: linbr@126.com

The GenBank accession numbers for $16 \mathrm{~S}$ rDNA, dnaX, gryB, and recA of Dickeya zeae MS1 are JQ301888, JQ284038, JQ284039, and KC793203, respectively.

The $\boldsymbol{e}$-Xtra logo stands for "electronic extra" and indicates that two supplementary figures are available online.

Accepted for publication 27 October 2014.

http://dx.doi.org/10.1094/PDIS-07-13-0711-RE

(C) 2014 The American Phytopathological Society obtained from infected plants from the previously mentioned locations, but it was difficult to determine the exact classification of the pathogen (17) because of the complex classification and taxonomic history of the genus Dickeya (24).

Since discovery of this disease in 2009 , its incidence and severity have increased. From 2010 to $2012,6,000$ ha of bananas were affected in Guangdong province. The mean disease incidence ranged from 20 to $70 \%$, but in some plantations incidences as high as $90 \%$ were observed. By 6 to 8 days after the first symptoms were observed, infected plants died. Therefore, the control of this disease including seedling quarantine is important for the Chinese banana industry. Identifying the species that causes banana soft rot in China is essential for effective quarantine and disease management. Traditional phytobacterial identification includes characterization of symptoms and biochemical properties (24), NGM medium (14), selective crystal violet polypectin medium (CVP) (9), and species-specific polymerase chain reaction (PCR). However, the PCR method used for detecting Dickeya sp. could not identify the pathogen at the species level, including primers for the pectinase genes pelADE (21), and pelZ (13). To identify the causal agent at the species level, representative strains were subjected to phenotypic assays and genetic analysis of multi-locus sites, as compared to known reference species of Dickeya sp.

\section{Materials and Methods}

Isolation of causal agent. In three plantations in Guangzhou, banana plants with soft rot symptoms were collected at different locations. The pseudostem bases of 65 infected plants were sampled and stored separately in an ice box, followed by immediate pathogen isolation. Direct isolation was performed on rotting pseudostem tissues from symptomatic plants. Pseudostem tissues from the margins between diseased and healthy areas were cut into 1.5 $\mathrm{cm}$ pieces and surface-sterilized by dipping in $75 \%$ ethanol for 30 $\mathrm{s}, 1 \% \mathrm{NaOCl}$ for $1 \mathrm{~min}$, and rinsing with sterile distilled water three times. The sterilized sections were macerated in drops of sterile water, and the tissue extract was streaked onto nutrient agar ( $3 \mathrm{~g} /$ liter beef extract, $0.5 \mathrm{~g} /$ liter yeast extract paste, $5 \mathrm{~g} /$ liter pep- 
tone, and $10 \mathrm{~g} /$ liter agar, $\mathrm{pH} 7.0$ ) and cultured for $48 \mathrm{~h}$ at $32^{\circ} \mathrm{C}$. Single colonies were chosen for subculturing three times to obtain strains.

Biochemical characterization. Forty-five strains from diseased plants were confirmed by Koch's postulates, and five of them were selected for biochemical characterization. The five strains were assessed for their ability to utilize 95 sugars, alcohols, acids, amines, and other substrates using the BIOLOG automated microbial analysis system (Version 4.20.05, Hayward, CA 94545 USA). One hundred fifty microliters of a bacterial suspensions containing $10^{8} \mathrm{CFU} / \mathrm{ml}$ of each strain was added to each well of a BIOLOG GN micro-plate according to the manufacturer's instruction.
Amplification and sequencing of $16 \mathrm{~S}$ rDNA, $\operatorname{dnaX}$, gyrB, and rec $A$ genes. Bacteria were grown at $30^{\circ} \mathrm{C}$ for $24 \mathrm{~h}$ in $\mathrm{LB}$ medium $(10 \mathrm{~g} /$ liter bacto tryptone, $5 \mathrm{~g} /$ liter yeast extract, and $10 \mathrm{~g} / \mathrm{liter}$ $\mathrm{NaCl}, \mathrm{pH} 7.0$ ) in an incubator shaker at $100 \mathrm{RPM}$. Total DNA was extracted from bacterial suspensions $\left(10^{8} \mathrm{CFU} / \mathrm{ml}\right)$ with a TIANamp Bacterial DNA kit (Tiangen Biotech, Beijing, China) according to manufacturer's directions. A $1,503 \mathrm{bp}$ region of the $16 \mathrm{~S}$ ribosomal DNA (rDNA) gene, $536 \mathrm{bp}$ of DNA polymerase III gamma subunit gene (dnaX), 2,412 bp of DNA gyrase gene $(\mathrm{gyr} B)$, and $700 \mathrm{bp}$ of recombinase $\mathrm{A}$ gene $(\mathrm{rec} A)$ were amplified using primer pairs fD2/rP1 (30), dnaXf/dnaXr (25), gyrBf1/gyrBr1 designed with published gyrB gene sequences of Dickeya genus in
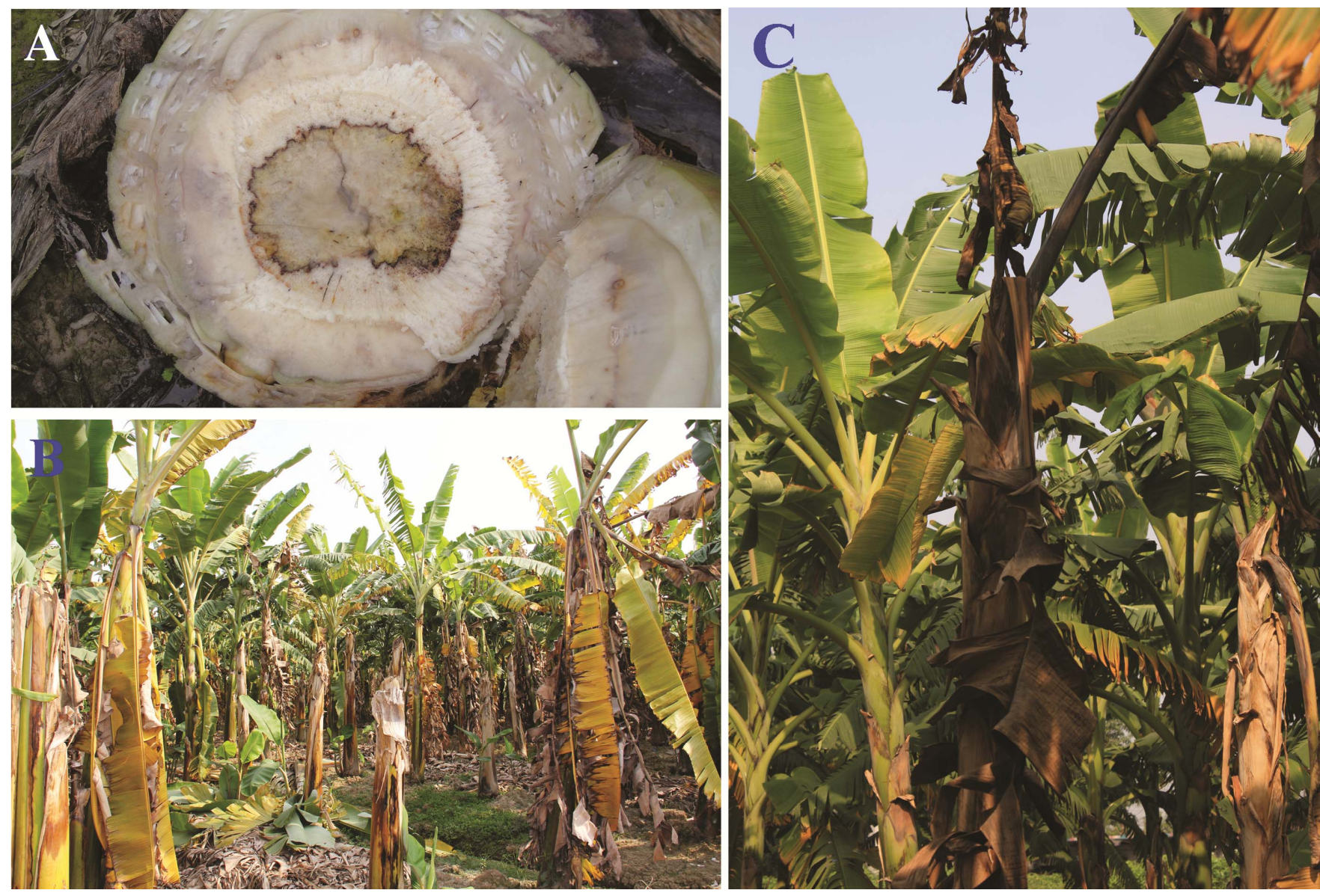

Fig. 1. Symptoms of bacterial soft rot of banana. A, Cross-sections of pseudostem of diseased banana; B, incidence of soft rot in a banana plantation; C, symptoms of a whole plant.
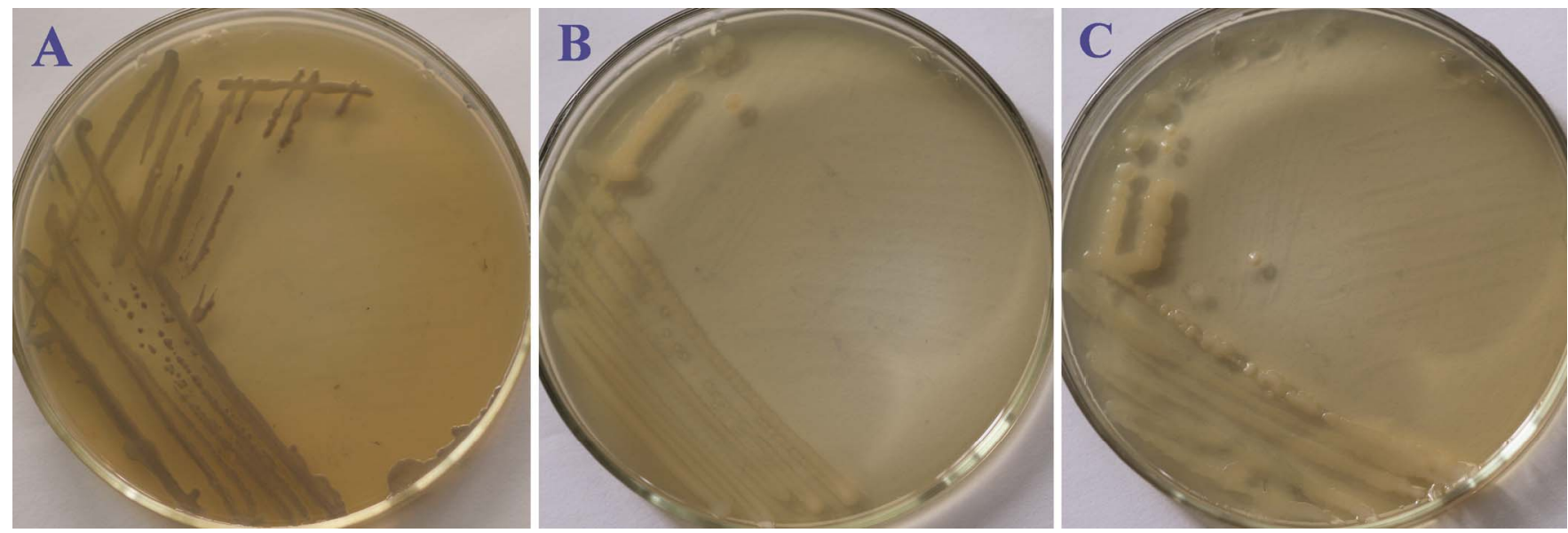

Fig. 2. Culturing characteristics of three Dickeya strains at $48 \mathrm{~h}$. MS1 produced gray colonies on nutrient agar compared to other strains. A, MS1 was isolated from banana; B, EC1 was D. zeae strain from rice; C, PA1 was Dickeya sp. strain from phalaenopsis. 
the National Center for Biotechnology Information (NCBI) database, and recAf/recAr (29), respectively (Table 1). For PCR amplification, $50 \mu \mathrm{l}$ of reaction mixtures contained 10x TransStart FastPfu Buffer, $250 \mu \mathrm{M}$ dNTPs, $0.4 \mu \mathrm{M}$ of each primer, $2.5 \mathrm{U}$ TransStart FastPfu DNA Polymerase (TransGen Biotech), and $2 \mu \mathrm{l}$ of template DNA. PCR reactions were performed using the following thermal profile: initial denaturation for $2 \mathrm{~min}$ at $95^{\circ} \mathrm{C}$, followed by 30 cycles of $94^{\circ} \mathrm{C}$ for $30 \mathrm{~s}, 55^{\circ} \mathrm{C}$ for $30 \mathrm{~s}$, and $72^{\circ} \mathrm{C}$ for $2 \mathrm{~min}$, followed by a final extension at $72^{\circ} \mathrm{C}$ for $7 \mathrm{~min}$. PCR products were separated on a $1 \%$ agarose gel and purified with a PCR purification kit (TransGen Biotech) according to the manufacturer's instructions. After purification, the products were cloned into the pEASY-T vector (TransGen Biotech); transformed into Escherichia coli $\mathrm{DH} 5 \alpha$ competent cells, and transformants were selected on LB agar amended with $100 \mu \mathrm{g} / \mathrm{ml}$ ampicillin and $50 \mu \mathrm{g} / \mathrm{ml}$ kanamycin. Transformants were validated by colony PCR using common primers M13F (5'-GTAAAACGACGGCCAGT-3') and M13R (5'-CAGGAAACAGCTATGAC-3'), and the positive clones were grown in $\mathrm{LB}$ at $37^{\circ} \mathrm{C}$ for $12 \mathrm{~h}$. Plasmids were extracted with a TIANprep Mini Plasmid kit (Tiangen Biotech) and sequenced in Sangon Co. (Shanghai, China).

Phylogenetic analyses. Each 16S rDNA, dnaX, gryB, and recA sequence was subjected to BLAST analysis against sequences in the National Center for Biotechnology Information (NCBI). Dickeya species with complete $\operatorname{dnaX}$, gryB, and recA sequences in NCBI GenBank were used for phylogenetic analyses, including 15 whole-genome sequenced Dickeya strains, of which six were type Dickeya strains in a previous study (22). A preliminary automatic alignment of the sequences was generated using CLUSTALW (8) with a gap penalty of 15 and then clipped to the length of consensus sequences. The concatenated, informative sequences of the $d n a X, \operatorname{gry} B$, and recA gene sequences were used for phylogenetic analysis using MEGA 5.1 (27) with a neighbor-joining method and bootstrapping test of 1,000 replicates.

Pathogenicity test. Pathogenicity tests were conducted by injecting $100-\mu$ bacterial suspensions $\left(10^{8} \mathrm{CFU} / \mathrm{ml}\right)$ of five representative strains of the pathogen into each of 10 banana seedlings (cultivar of Musa Cavendish var. Brazil) at the trefoil stage. Ten banana seedlings inoculated with sterile water served as negative controls. Plants were placed in a greenhouse at $30^{\circ} \mathrm{C}$ and $90 \%$ relative humidity for 1 week. The seedlings showing soft rot symptoms were photographed and used for bacterial reisolation. The strains were reisolated as described above, and PCR assays using primer pairs $\mathrm{fD} 2 / \mathrm{rP} 1$ for $16 \mathrm{~S}$ rDNA were performed for pathogen confirmation. 16S rDNA sequence data from the reisolated strains were compared to those of the original strains isolated from banana. In another case, banana leaves were detached from seedlings at the five- or six-leaf stage. Entire carpopodiums filled with immature fruits were also detached from the plants and kept at high relative humidity for the pathogenicity test. Fifty microliters of bacterial suspension $\left(10^{8} \mathrm{CFU} / \mathrm{ml}\right)$ of five representative strains was injected into each banana leaf and fruit, and injections of sterile water served as negative control treatments. The injected leaves and fruits were incubated at $30^{\circ} \mathrm{C}$, and the injection sites were wrapped with wet cotton balls moisturized with sterile water.

Pathogenicity of the strains was confirmed on seedlings of tobacco, maize, rice, and sugarcane, and the potato tubers, cucumber, tomato, watermelon, Chinese cabbage, towel gourd, onion, carrot, papaya, apple, and pineapple. Inoculation was conducted as described above for banana leaves and fruits.

Prevalence survey. Between September and November 2010, banana plantations were surveyed for prevalence of soft rot in Guangdong province (Panyu district and Nansha district of Guangzhou city, Dongguan city, Zhongshan city, Zhuhai city, Gaozhou city, Huazhou city, Laozhou island, Donghai island, and Xuwen county of Zhanjiang city, Jieyang city, and Shantou city), Guangxi province (Nanning city), Fujian province (Zhangzhou city), Hainan province (ChengMai county, Lingao county, Ledong county, Dongfang city, and Chenwang county). Banana plants with soft rot symptoms were collected and used for pathogen isolation and characterization. Plant tissues were assayed directly by PCR using ITS primers LF/LR (LF 5'-TTCGTCTAGAGGCCCAGGAC-3' and LR 5'-TCAGCTTGTTCCGGATTGTT-3') (16), and bacteria were isolated as describe above.

\section{Results}

Characterization of $D$. zeae strains. Strains from diseased bananas produced round or nearly round colonies on nutrient agar that were slightly convex, opaque, wrinkled, and dull. When cultured at $32^{\circ} \mathrm{C}$ for 24 to $48 \mathrm{~h}$, the colonies appeared gray (Fig. 2). Over time, the gray pigment disappeared, and the bacterium did not produce the gray pigment with subsequent subcultures.

Five representative strains were selected for biochemical analysis. They displayed pectinolytic activity, production of indole, and grew at $39^{\circ} \mathrm{C}$. BIOLOG analysis identified the strains as Pectobacterium chrysanthemi, and the five strains showed the highest similarities of $0.638,0.718,0.729,0.718$, and 0.523 with $P$. chrysanthemi. The five strains from banana catabolized L-arabinose,

Table 1. Polymerase chain reaction (PCR) primer sequences used to analyze banana soft rot strains collected in China and corresponding predicted amplicon sizes

\begin{tabular}{|c|c|c|c|c|}
\hline Locus & Primers & Sequence & Product size (bp) & Reference \\
\hline 16S rDNA & fD2 & 5'-AGAGTTTGATCATGGCTCAG-3' & 1,503 & Weisburg et al. (30) \\
\hline & rP1 & 5'-GGTTACCTTGTTACGACTTC- $3^{\prime}$ & & \\
\hline dnaX & dnaXf & 5'-TATCAGGTYCTTGCCCGTAAGTGG-3' & 536 & Slawiak et al. (25) \\
\hline & dnaXr & 5'-TCGACATCCARCGCYTTGAGATG-3' & & \\
\hline gyrB & $\begin{array}{l}\text { gyrBf1 } \\
\text { gyrBr1 }\end{array}$ & $\begin{array}{l}5^{\prime} \text {-ATGTCGAATTCTTATGACTCCTC-3' } \\
5^{\prime} \text {-TCARATATCRATATTCGCYGCTTTC-3' }\end{array}$ & 2,412 & \\
\hline recA & recAf & $\begin{array}{l}5^{\prime} \text {-GGTAAAGGGTCTATCATGCG-3' } \\
5^{\prime} \text {-CCTTCACCATACATAATTTGGA-3' }\end{array}$ & 700 & Waleron et al. (29) \\
\hline
\end{tabular}

Table 2. Different characteristics of six Dickeya species $^{\mathrm{a}}$ and the banana soft rot pathogens isolated in China ${ }^{\mathrm{b}}$

\begin{tabular}{lccccc}
\hline Characteristics & Growth at $\mathbf{3 9}^{\circ} \mathbf{C}$ & Myo-inositol & cis-Aconitete & D-Melibiose & Mannitol \\
\hline D. dadantii + D. zeae & + & + & + & + & + \\
D. chrysanthemi bv. parthenii & + & + & - & + & + \\
D. chrysanthemi bv. chrysanthemi & + & + & $\mathrm{P}$ & - & + \\
D. dieffenbachiae & + & + & - & $\mathrm{P}$ & + \\
D. dianthicola & - & + & - & $\mathrm{P}$ & + \\
D. paradisiaca & + & + & + & + \\
Dickeya sp. from banana & + & + & & + \\
\hline
\end{tabular}

a Data of six Dickeya species referred to Samson et al. (24).

$\mathrm{b}+$ Indicated positive, - indicated negative, and $\mathrm{P}$ indicated partially positive. 
myo-inositol, D-mannose, cis-aconitate, D-melibiose, and mannitol, but failed to utilize D-trehalose, D-arabitol, $\beta$-gentiobiose, and sorbitol. Compared to the known characteristics of six Dickeya species including $D$. zeae, $D$. dadantii, D. paradisiaca, D. chrysanthemi, $D$. dieffenbachiae, and $D$. dianthicola, the banana pathogen and D. dadantii or D. zeae grew at $39^{\circ} \mathrm{C}$, catabolized myo-inositol, cis-aconitate, D-melibiose, and mannitol, while D. paradisiaca did not grow at $39^{\circ} \mathrm{C}$ nor use these four carbon sources. D. chrysanthemi, $D$. dieffenbachiae, and $D$. dianthicola also differed by one, two, and three characteristics with the banana pathogen, respectively (Table 2). The phenotypic characteristics of the banana pathogen were most consistent with $D$. dadantii and D. zeae.

Phylogenetic analysis based on housekeeping genes. One typical strain of the five banana strains, MS1, was used for genetic analysis, and the sequence data for four conserved genes were deposited in GenBank (GenBank accession numbers JQ301888 for $16 \mathrm{~S}$ rDNA, JQ284038 for $\operatorname{dnaX}$, JQ284039 for $\operatorname{gryB}$, and KC793203 for recA). BLAST analysis indicated that all of these sequences were $99 \%$ similar to the sequences of D. zeae.

Twenty-eight strains of different Dickeya species with complete sequences of $\operatorname{dnaX}, \operatorname{gryB}$, and $\operatorname{rec} A$ in NCBI GenBank were retrieved for analysis, along with an out group control, Pectobacterium carotovorum subsp. carotovorum $\mathrm{PC} 1$. The combined sequences of these three conserved genes of Dickeya strains consisted of 1,715 nucleotides (Supplementary Figure 1), of which 1,305 were constant, 410 were variable (388 were parsimony informative), and 22 were singleton. The phylogenetic analysis of concatenated sequences yielded six well-separated clades in the subgroup of Dickeya sp. (Fig. 3), and all the clades represented respective Dickeya species with each type strain, of which D. para- disiaca clade was distinct from the other five species. In the $D$. zeae clade, MS1 clustered with D. zeae NCPPB3532, D. zeae NCPPB2538, D. zeae EC1, and D. zeae ZJU1202; strains EC1 and ZJU1202 were isolated from rice in Guangdong province. They formed a strongly supported subclade with $100 \%$ bootstrap values, which was significantly different from other Dickeya species. The phylogenies strongly supported the lineages of each gene leading to the classification of the banana pathogen as D. zeae.

Pathogenicity studies of $D$. zeae from banana. All five strains of the banana pathogen caused vascular tissue collapse or parenchyma necrosis. Banana leaves, fruits, and seedlings showed soft rot symptoms within 48 to $72 \mathrm{~h}$ of inoculation with bacterial strains isolated from banana. Negative control plants did not develop symptoms. The injection of bacterial suspensions into leaf veins caused the leaves to appear water-soaked and wilted. The injection of bacterial suspensions into the stem base resulted in rotting of the whole plant and emission of a foul odor. Fruit inoculation caused the internal fruit rot. The symptoms caused by MS1 are shown in Figure 4A, B, and C. The results for other strains were similar; therefore, their data were not presented. D. zeae was reisolated from infected seedlings and confirmed to be identical to the original pathogen based on $16 \mathrm{~S}$ rDNA sequence comparison.

Dickeya species have a wide host range and induce soft rot on different plants species. D. zeae infected most of the plant species that were inoculated, including potato (Fig. 4D), cucumber (Fig. 4E), tomato (Fig. 4F), watermelon, cabbage (Fig. 4G), onion, carrot (Fig. 4H), towel gourd (Fig. 4I), tobacco, rice, maize, and sugarcane. In most cases, the rot was accompanied by a sharp odor. Moreover, inoculated rice plants showed severe foot rot symptoms 1 week after inoculation (Fig. 4J). Inoculated corn and sugarcane

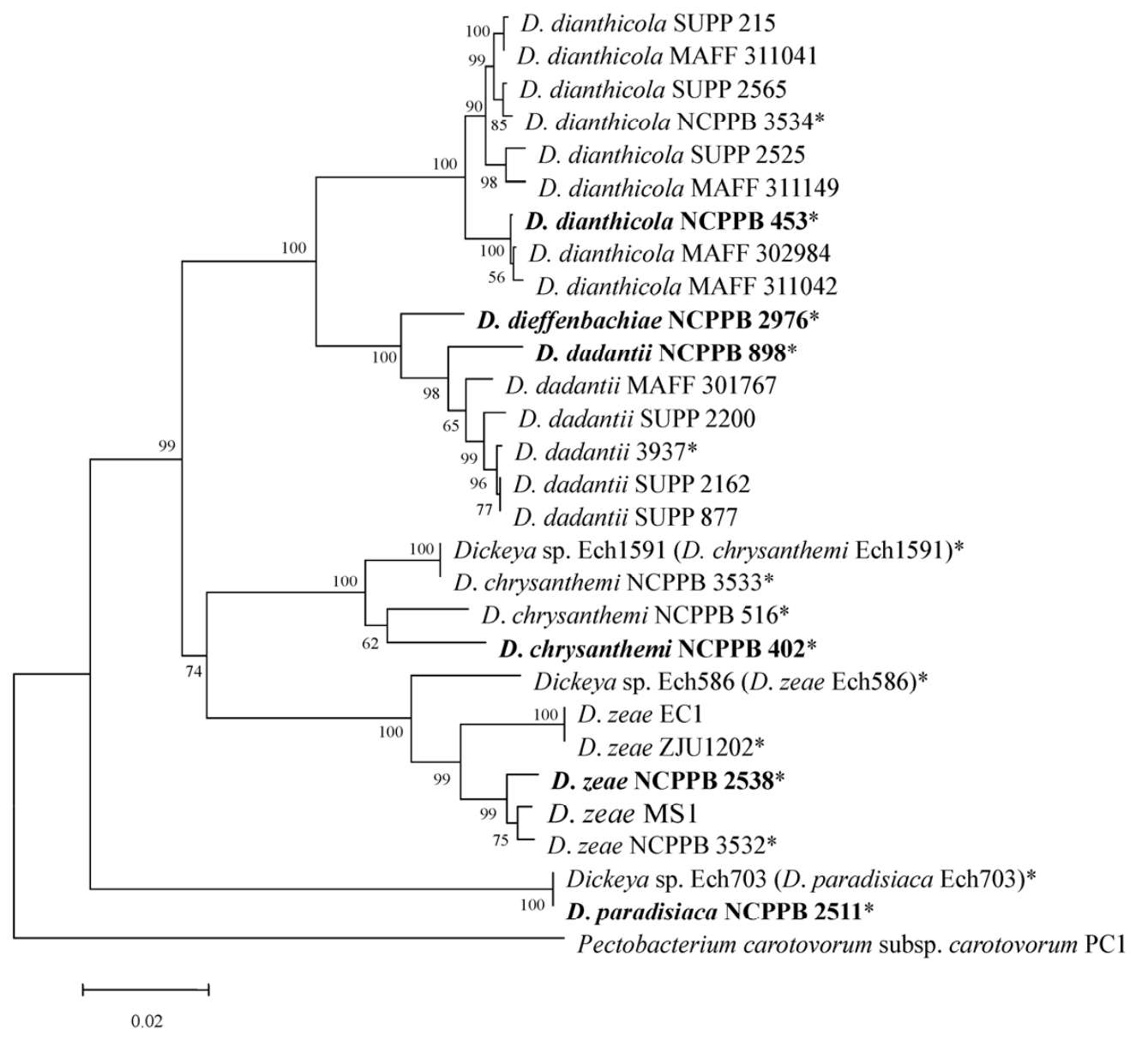

Fig. 3. Phylogenetic analysis of MS1 and Dickeya strains based on concatenated sequences of $d n a X$, gryB, and recA genes. Confidence values on the branches were obtained for 1,000 bootstrap replicates with Mega version 5.1 software. Twenty-eight Dickeya strains were used for phylogenetic analyses, and the sequences of 15 strains were retrieved from whole-genome sequences as indicated with asterisks. Type Dickeya strains (22) are shown in boldface. D. dadantii Ech586, D. zeae Ech1591, and D. dadantii Ech703 were suggested to be reclassified as D. zeae Ech586, D. chrysanthemi Ech1591, and D. paradisiaca Ech503, respectively (19). 
seedlings showed stalk rot or foot rot symptoms. However, the pathogen did not induce soft rot on papaya, apple, and pineapple fruits (Table 3).

Disease prevalence in mainland China. Banana soft rot was first observed in September 2009 in Guangzhou city, and spread rapidly through the banana-growing regions of China. The disease usually had a peak incidence between September and November. Sixty-four symptomatic banana plants were sampled from 16 locations of south China. Of these, Dickeya strains were detected in 56 plants by PCR using specific ITS primers (Table 4). Thirty-nine
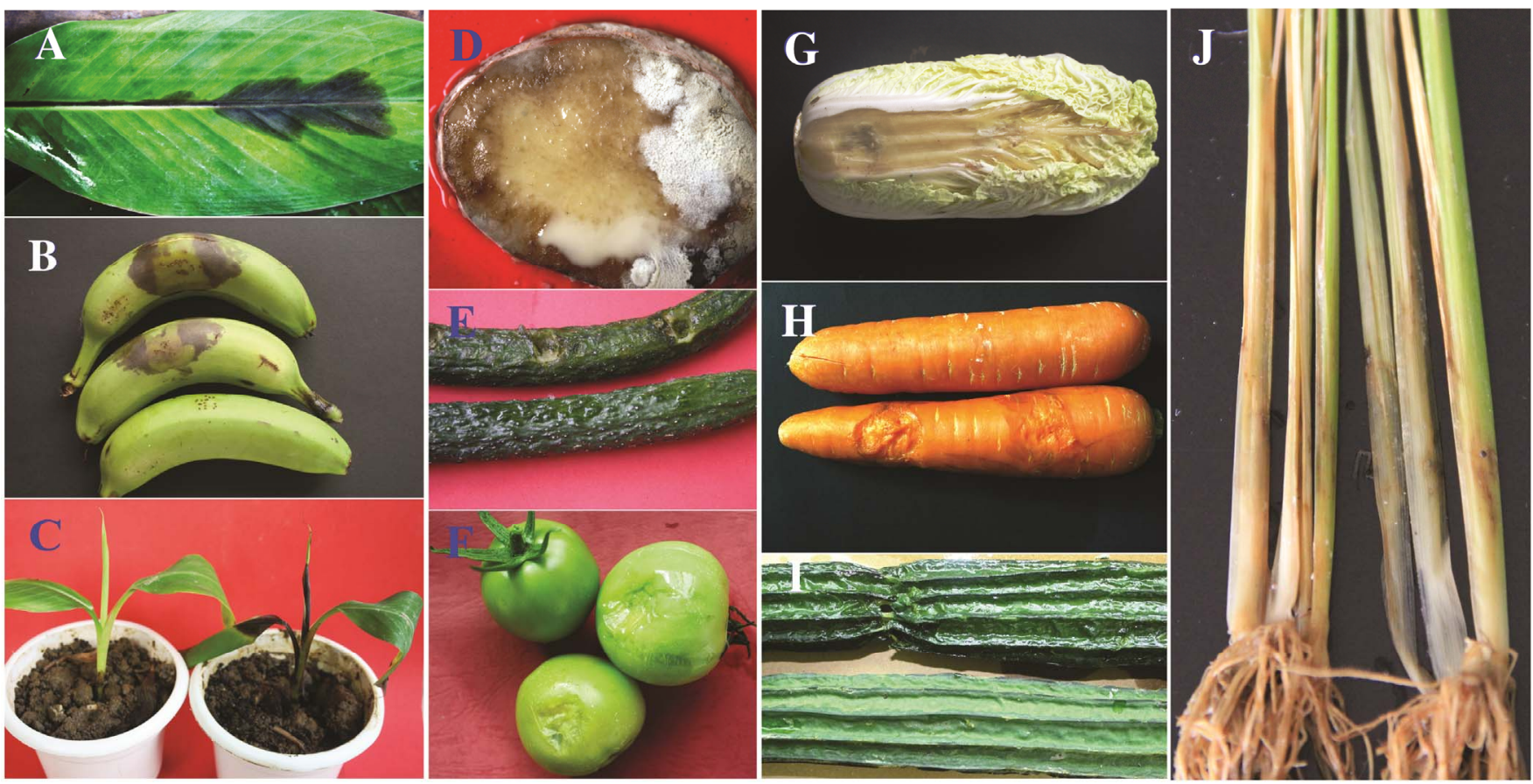

Fig. 4. Symptoms of soft rot of different crops after inoculation with bacterial suspensions of MS1. A, Banana leaf $48 \mathrm{~h}$ after inoculation; B, banana fruits $48 \mathrm{~h}$ after inoculation; C, banana seedlings $72 \mathrm{~h}$ after inoculation; D, potato slices $48 \mathrm{~h}$ after inoculation; E, cucumbers $24 \mathrm{~h}$ after inoculation; F, tomatoes $16 \mathrm{~h}$ after inoculation; G, Chinese cabbages $48 \mathrm{~h}$ after inoculation; H, carrots $24 \mathrm{~h}$ after inoculation; I, towel gourds $24 \mathrm{~h}$ after inoculation; J, rice seedlings a week after inoculation. Uninfected materials were inoculated with sterile water as controls.

Table 3. Pathogenicity of the banana soft rot pathogen Dickeya zeae MS1 on various plant species ${ }^{\mathrm{a}}$

\begin{tabular}{lccccc}
\hline Plant & Pathogenicity & Plant & Pathogenicity & Plant & Pathogenicity \\
\hline Potato tube & + & Towel gourd & + & Carrot & + \\
Cucumber & + & Onion & + & Papaya & - \\
Tomato & + & Tobacco & Pineapple & - \\
Watermelon & + & Rice & + & Sugarcane & + \\
Chinese cabbage & + & Maize & + & Apple & - \\
\hline
\end{tabular}

${ }^{a}+$ Indicated positive, - indicated negative.

Table 4. Isolation and polymerase chain reaction (PCR) validation of strains of the banana soft rot pathogen from various locations in China

\begin{tabular}{|c|c|c|c|c|c|c|}
\hline Location & Cultivar & Gene type & Date & $\begin{array}{l}\text { Quantity } \\
\text { of samples }\end{array}$ & $\begin{array}{c}\text { Positive by } \\
\text { PCR detection }\end{array}$ & $\begin{array}{c}\text { Positive by } \\
\text { pathogen isolation }\end{array}$ \\
\hline Dongguan Guangdong & Musa Cavendish var. Brazil & AAA & 2010.09 .08 & 3 & 3 & 2 \\
\hline Panyu Guangzhou Guangdong & Guangfen-1 (dwarf banana) & $\mathrm{ABB}$ & 2010.09 .15 & 2 & 2 & 2 \\
\hline Nansha Guangzhou Guangdong & Guangfen-1 (dwarf banana) & $\mathrm{ABB}$ & 2010.09 .22 & 5 & 4 & 3 \\
\hline Zhongshan Guangdong & Guangfen-1 (dwarf banana) & $\mathrm{ABB}$ & 2010.09 .28 & 2 & 2 & 2 \\
\hline Zhuhai Guangdong & Guangfen-1 (dwarf banana) & $\mathrm{ABB}$ & 2010.10 .08 & 3 & 3 & 2 \\
\hline Jieyang Guangdong & Musa Cavendish var. Brazil & AAA & 2010.10 .08 & 1 & 1 & 1 \\
\hline Shantou Guangdong & Musa Cavendish var. Brazil & AAA & 2010.10 .08 & 1 & 1 & 0 \\
\hline Gaozhou Guangdong & Musa Cavendish var. Brazil & AAA & 2010.10 .28 & 3 & 3 & 2 \\
\hline Huazhou Guangdong & Musa Cavendish var. Brazil & AAA & 2010.10 .28 & 2 & 2 & 0 \\
\hline $\begin{array}{l}\text { Zhanjiang Laozhou Island } \\
\text { Guangdong }\end{array}$ & Musa Cavendish var. Brazil & AAA & 2010.10 .28 & 8 & 7 & 4 \\
\hline Xuwen Guangdong & Musa Cavendish var. Brazil & AAA & 2010.10 .28 & 3 & 3 & 3 \\
\hline Zhangzhou Fujian & Tianbao & AAA & 2010.10 .20 & 8 & 7 & 4 \\
\hline Zhangzhou Fujian & Musa Cavendish var. B. F. & AAA & 2010.10 .20 & 3 & 3 & 1 \\
\hline Dongfang Hainan & Musa Cavendish var. Brazil & AAA & 2010.11 .3 & 9 & 6 & 5 \\
\hline Chenmai Hainan & Musa Cavendish var. Brazil & AAA & 2010.11 .3 & 5 & 3 & 3 \\
\hline Chenwang Hainan & Unnamed dwarf banana & $\mathrm{ABB}$ & 2010.11 .3 & 2 & 2 & 2 \\
\hline Nanning Guangxi & Guangfen-1 (dwarf banana) & $\mathrm{ABB}$ & 2010.11 .15 & 4 & 4 & 3 \\
\hline Total & & & & 64 & 56 & 39 \\
\hline
\end{tabular}

a All banana plants from which Dickeya strains could be isolated were detected as positive samples through PCR. 
strains were isolated from these diseased plants, and all strains produced colonies with specific gray pigment that distinguished them from other Dickeya species. The disease was present in most of the surveyed locations, including Dongguan, Panyu Guangzhou, Nansha Guangzhou, Zhongshan, Zhuhai, Jieyang, Gaozhou, Laozhou island, Zhanjiang, and Xuwen of Guangdong province, Zhangzhou of Fujian province, Nanning of Guangxi province, and Dongfang, Chenmai, and Chenwang of Hainan province. The disease affected different banana cultivars, including AAA type, Musa Cavendish var. Brazil, local cultivars Tianbao (from Fujian) and Musa Cavendish var. B. F. (from Taiwang), ABB type, dwarf cultivar Guangfen-1 (from Guangzhou), and an unnamed dwarf banana cultivar (from Hainan).

\section{Discussion}

Dickeya sp., formerly named Erwinia chrysanthemi and Pectobacterium chrysanthemi (7), is a recently established genus (24) that includes necrotrophic pathogens responsible for soft rot in a wide range of hosts (18). According to host range assays, biochemical characteristics, phylogenetic analyses, and serological reactions, these gram-negative pathogens were divided into six species: D. chrysanthemi, $D$. dianthicola, D. dieffenbachiae, D. paradisiaca, $D$. dadantii, and $D$. zeae $(4,24)$. However, there was no diagnostic test that allows accurate clarification of taxonomy until now (24). Since different species are closely related, or lack strains with standard biochemical or genetic characteristics, it is not sufficient to distinguish them based on biochemical reactions alone or single gene sequences analysis $(12,19)$. Therefore, this study combined biochemical characteristics and multi-gene sequence analysis to determine the identity of the banana pathogen, because multi-gene sequence analysis can provide a better understanding of phylogeny information in bacteria (5). In this study, we analyzed the sequences of four genes including $16 \mathrm{~S}$ rDNA, dnaX, gyrB, and recA to identify the banana pathogen. For Dickeya spp., dnaX gene sequences were used as identification tags for the classification of complex biovars (25). The $g y r B$ gene is a recommended identification tag to describe the phylogenetic relationships among Enterobacteriaceae (2). The gyrB gene can also distinguish different strains at the species level $(10,11,31)$; therefore, we designed a set of PCR primers to amplify the gyrB gene for the banana strains. The phylogenetic analysis based on recA sequences also provides an effective method for determining Dickeya strains to the species and intraspecies level (22). In virtue of multi-locus sequence analysis (MLSA) of $d n a A, \operatorname{gyr} B$, and $r e c A$, different Dickeya strains formed six distinct subclades representing respective Dickeya species, supported with each type strain. However, it was also noteworthy that $D$. dadantii Ech586, D. zeae Ech1591, and D. dadantii Ech703 were not placed in their respective clades. The same conclusion was also illustrated in a previous study (19), and they were recommended to be reclassified as $D$. zeae Ech586, D. chrysanthemi Ech1591, and D. paradisiaca Ech503, respectively.

Through the use of biochemical characteristics and phylogenetic analysis of multi-locus sequences, the pathogen isolated from banana was determined to be $D$. zeae. First, the pathogen showed the most similar biochemical characteristics to $D$. zeae and $D$. dadantii, and differed in several characteristics from other species, especially $D$. paradisiaca. Dickeya species (E. chrysanthemi) that cause bacterial soft rot on banana were usually reported to be $D$. paradisiaca, as initially observed in Columbia $(3,24)$. However, the strain affecting banana in Guangdong differed from the typical $D$. paradisiaca strains as indicated by biochemical characteristics and sequence analysis of conserved genes. According to BLAST analysis, D. paradisiaca did not appear in the list of the top 100 most similar sequences. Secondly, the banana strain clustered with $D$. zeae $\mathrm{EC1}$ (23) and D. zeae ZJU1202 (15), both of which were isolated from rice in Guangdong province, and formed a distinguishable clade based on concatenated sequences of $\operatorname{dnaX}$, gry $B$, and recA. Interestingly, $D$. zeae from banana had some unique properties compared to $D$. zeae from rice in Guangdong province, indicating that it is a specific Dickeya strain. For example, it has gray colonies (Fig. 2A), prefers anaerobic conditions, and lacks phytotoxins. The phytotoxin gene cluster, $z m s A$, was not detected in D. zeae MS1 using specific primers (32), and it did not produce an antibiotic effect against $E$. coli on LB medium (Supplementary Figure 2), while EC1 inhibited the growth of E. coli (32). The pathogenicity of MS1 is different from the rice strains, the diversity of strains colonizing different hosts. The difference between the banana and rice strains was also supported by grouping of the concatenated sequences. The banana strain and other D. zeae strains comprised a clade that was separate from D. zeae EC1, ZJU1202.

D. zeae has been shown to cause soft rot of banana in Columbia, Ivory Coast, Panama, and several Caribbean islands (24), but to our knowledge, this is the first report of banana soft rot caused by $D$. zeae in China. Recently, the disease has been found in most banana-growing areas in China, and our results indicate the causal pathogen has many potential hosts in the grasses, Crucifer, Solanaceae, Cucurbitaceae, and Liliaceae, which are important crops. Therefore, the pathogen should be studied to develop effective management strategies. Our findings will help to develop molecular tools for detection and ultimately effective control strategies for bacterial soft rot of banana.

\section{Acknowledgments}

This work was granted by the International Cooperation Projects of Science and Technology of Guangdong Province (2012B050500014), Guangdong Science and Technology Project (2011B031500030), and Guangzhou Science and Technology Project (12C14071616).

\section{Literature Cited}

1. Chio, J. E., Park, J. S., and Kang, H. W. 1988. Bacterial soft rot of banana fruit caused by Erwinia carotovora subsp. carotovora and Pseudomonas cichorii. Korean J. Plant Pathol. 3:202-206.

2. Dauga, C. 2002. Evolution of the gyrB gene and the molecular phylogeny of Enterobacteriaceae: A model molecule for molecular systematic studies. Int. J. Syst. Evol. Microbiol. 52:531-547.

3. Dickeya, R. S., and Victoria, J. I. 1980. Taxonomy and emended description of strains of Erwinia isolated from Musa paradisiaca Linnaeus. Int. J. Syst. Evol. Microbiol. 30:129-134.

4. Dye, D. W., Bradbury, J. F., Goto, M., Hayward, A. C., Lelliott, R. A., and Schroth, M. N. 1980. International standards for naming pathovars of phytopathogenic bacteria and a list of pathovar names and pathotype strains. Rev. Plant Pathol. 59:153-168.

5. Hanage, P. W., Fraser, C., and Spratt, B. G. 2006. Sequences, sequence clusters and bacterial species. Philos. Transact. Roy. Soc. 361:1917-1927.

6. Hassanzadeh, N. 1990. Characterization of a new soft rot Erwinia to banana in Iran. Iranian J. Plant Pathol. 26:13-18.

7. Hauben, L., Moore, E. R. B., Vauterin, L., Steenackers, M., Mergaert, J., Verdonck, L., and Swings, J. 1998. Phylogenetic position of phytopathogens within the Enterobacteriaceae. Syst. Appl. Microbiol. 21:384-397.

8. Higgins, D., Thompson, J., Gibson, T., Thompson, J. D., Higgins, D. G., and Gibson, T. J. 1994. CLUSTAL W: Improving the sensitivity of progressive multiple sequence alignment through sequence weighting, positionspecific gap penalties and weight matrix choice. Nucleic Acids Res. 22:4673-4680.

9. Hyman, L. J., Sullivan, L., Toth, I. K., and Perombelon, M. C. M. 2001 Modified crystal violet pectate medium (CVP) based on a new polypectate source (Slendid) for the detection and isolation of soft rot Erwinias. Potato Res. 44:265-270.

10. Kasai, H., Ezak, T., and Harayama, S. 2000. Differentiation of phylogenetically related slowly growing mycobacteria by their $\operatorname{gyr} B$ sequences. J. Clin. Microbiol. 38:301-308.

11. Kasai, H., Tamura, T., and Harayama, S. 2000. Intrageneric relationships among Micromonoopora species deduced from gyrB-based phylogeny and DNA relatedness. Int. J. Syst. Evol. Microbiol. 50:127-134.

12. Kolbert, C. P., and Persing, D. H. 1999. Ribosomal DNA sequencing as a tool for identification of bacterial pathogens. Curr. Opin. Microbiol. 2:299305 .

13. Lee, Y. A., Chen, K. P., and Hsu, Y. W. 2006. Characterization of Erwinia chrysanthemi, the soft-rot pathogen of white-flowered calla lily, based on pathogenicity and PCR-RFLP and PFGE analyses. Plant Pathol. 55:530536.

14. Lee, Y. A., and Yu, C. P. 2006. A differential medium for the isolation and rapid identification of a plant soft rot pathogen, Erwinia chrysanthemi. J. Microbiol. Methods 64:200-206.

15. Li, B., Shi, Y., Ibrahim, M., Liu, H., Shan, C. L., Wang, Y. L., Kube, M., Xie, G. L., and Sun, G. C. 2012. Genome sequence of the rice pathogen Dickeya zeae strain ZJU1202. J. Bacteriol. 194:4452-4453. 
16. Li, P. Q., Lin, B. R., Shen, H. F., and Pu, X. M. 2011. Species-specific detection of Dickeya sp. (Pectobacterium chrysanthemi) in infected banana tissues, soil and water. Afr. J. Biotechnol. 10:16774-16780.

17. Lin, B. R., Shen, H. F., Pu, X. M., Tian, X. S., Zhao, W. J., Zhu, S. F., and Dong, M. M. 2010. First report of a soft rot of banana in Mainland China caused by a Dickeya sp. (Pectobacterium chrysanthemi). Plant Dis. 94:640.

18. Ma, B., Hibbing, M. E., Kim, H. S., Reedy, R. M., Yedidia, I., Breuer, J., Glasner, J. D., Perna, N. T., Kelman, A., and Charkowski, A. O. 2007. Host range and molecular phylogenies of the soft rot enterobacterial genera Pectobacterium and Dickeya. Phytopathology 97:1150-1163.

19. Marrero, G., Schneider, K. L., Jenkins, D. M., and Alvarez, A. M. 2013. Phylogeny and classification of Dickeya based on multilocus sequence analysis. Int. J. Syst. Evol. Microbiol. 63:3524-3539.

20. Nabhan, S., Wydra, K., Linde, M., and Debener, T. 2012. The use of two complementary DNA assays, AFLP and MLSA, for epidemic and phylogenetic studies of pectolytic enterobacterial strains with focus on the heterogeneous species Pectobacterium carotovorum. Plant Pathol. 61:498-508.

21. Nassar, A., Darrasse, A., Lematter, M., Kotoujansky, A., Dervin, C., Vedel, R., and Bertheau, Y. 1996. Characterization of Erwinia chrysanthemi by pectinolytic isozyme polymorphism and restriction fragment length polymorphism analysis of PCR-amplified fragments of pel genes. Appl. Environ. Microbiol. 62: 2228-2235.

22. Parkinson, N., Stead, D., Bew, J., Heeney, J., Tsror, L., and Elphinstone, J. 2009. Dickeya species relatedness and clade structure determined by comparison of recA sequences. Int. J. Syst. Evol. Microbiol. 59:2388-2393.

23. $\mathrm{Pu}$, X. M., Zhou, J. N., Lin, B. R., and Shen, H. F. 2012. First report of Bacterial foot rot of rice caused by a Dickeya zeae in China. Plant Dis. 96:1818.

24. Samson, R., Legendre, J. B., Christen, R., Achouak, W., and Gardan, L. 2005. Transfer of Pectobecterium chrysanthemi (Burkholder et al., 1953) Brenner et al. 1973 and Brenneria paradisiaca to the genus Dickeya gen. nov. as Dickeya chrysanthemi comb. nov. and Dickeya paradisiaca comb. nov. and delineation of four novel species, Dickeya dadantii sp. nov., Dickeya dianthicola sp. nov., Dickeya dieffenbachiae sp. nov. and Dickeya zeae sp. nov. Int. J. Syst. Evol. Microbiol. 55:1415-1427.

25. Slawiak, M., van Beckhoven, J. R. C. M., Speksnijder, A. G. C. L., Czajkowski, R., Grabe, G., and van der Wolf, J. M. 2009. Biochemical and genetical analysis reveal a new clade of biovar 3 Dickeya spp. strains isolated from potato in Europe. Eur. J. Plant Pathol. 125:245-261.

26. Snehalatharani, A., and Khan, A. N. 2010. Biochemical and physiological characterization of Erwinia species causing tip-over disease of banana Arch. Phytopathol. Plant Prot. 43:1072-1080.

27. Tamura, K., Peterson, D., Peterson, N., Stecher, G., Nei, M., and Kumar, S 2011. MEGA5: Molecular evolutionary genetics analysis using maximum likelihood, evolutionary distance, and maximum parsimony methods. Mol. Biol. Evol. 28:2731-2739.

28. Thomas, P., Goplakrishnan, C., and Krishnareddy, M. 2011. Soft rot inciting: Pectobacterium carotovorum (Erwinia carotovora) is unlikely to be transmitted as a latent pathogen in micropropagated banana. Plant Cell Tissue Organ Cult. 105:423-429.

29. Waleron, M., Waleron, K., Podhajska, A., and Lojkowka, E. 2002. Genotyping of bacteria belonging to the former Erwinia genus by PCR-RFLP analysis of a recA gene fragment. Microbiology 148:583-595.

30. Weisburg, W. G., Barns, S. M., Pelletier, D. A., and Lane, D. J. 1991. 16S ribosomal DNA amplification for phylogenetic study. J. Bacteriol. 173:697703.

31. Yamamoto, S., and Harayama, S. 1996. Phylogenetic analysis of Acinetobacter strains based on the nucleotide sequences of $\mathrm{gyrB}$ genes and on the amino acid sequences of their products. Int. J. Syst. Evol. Microbiol. 46:506-511.

32. Zhou, J. N., Zhang, H. B., Wu, J., Liu, Q. G., Xi, P. G., Lee, J., Liao, J. L., Jian, Z. D., and Zhang, L. H. 2011. A novel multidomain polyketide synthase is essential for zeamine production and the virulence of Dickeya zeae. Mol. Plant-Microbe Interact. 24:1156-1164. 